Research Article

\title{
Some combinatorial identities concerning harmonic numbers and binomial coefficients
}

\section{Dongwei Guo*}

School of Mathematics and Statistics, Zhoukou Normal University, Henan, China

(Received: 6 September 2021. Received in revised form: 8 October 2021. Accepted: 21 October 2021. Published online: 25 October 2021.)

(c) 2021 the author. This is an open access article under the CC BY (International 4.0) license (www.creativecommons.org/licenses/by/4.0/).

\section{Abstract}

An elementary proof for a binomial identity of Batir [Integers 20 (2020) \#A25] is presented and several combinatorial identities concerning harmonic numbers and binomial coefficients are derived

Keywords: harmonic numbers; binomial coefficients; combinatorial identities.

2020 Mathematics Subject Classification: 05A19, 11B65.

\section{Introduction}

It is well-known that the harmonic numbers are defined by

$$
H_{0}=0 \quad \text { and } \quad H_{n}=\sum_{k=1}^{n} \frac{1}{k} \quad \text { for } \quad n \in \mathbb{N} .
$$

For $n, \ell \in \mathbb{N}$ and for an indeterminate $x$, they are generalized (cf. $[3,6])$ by

$$
H_{n}^{\langle\ell\rangle}(x)=\sum_{k=1}^{n} \frac{1}{(k+x)^{\ell}} \quad \text { and } \quad H_{n}^{\langle\ell\rangle}=H_{n}^{\langle\ell\rangle}(0)=\sum_{k=1}^{n} \frac{1}{k^{\ell}},
$$

which reduce, when $\ell=1$, to $H_{n}(x)$ (cf. $[4,7,8]$ ) and to the ordinary harmonic numbers $H_{n}$, respectively. Here and forth, we use $\mathbb{N}, \mathbb{Z}^{-}$and $\mathbb{C}$ to denote the sets of positive integers, negative integers and complex numbers, respectively.

Given a differentiable function $f(x)$, denote two derivative operators by

$$
\mathcal{D}_{x} f(x)=\frac{d}{d x} f(x) \quad \text { and } \quad \mathcal{D}_{0} f(x)=\left.\frac{d}{d x} f(x)\right|_{x=0} .
$$

Then it is not difficult to get the derivative of binomial coefficients (cf. [5, 6])

$$
\mathcal{D}_{x}\left(\begin{array}{c}
x+n \\
m
\end{array}\right)=\left(\begin{array}{c}
x+n \\
m
\end{array}\right) \sum_{\ell=1}^{m} \frac{1}{1+x+n-\ell},
$$

which can be stated in terms of the generalized harmonic numbers as

$$
\mathcal{D}_{x}\left(\begin{array}{c}
x+n \\
m
\end{array}\right)=\left(\begin{array}{c}
x+n \\
m
\end{array}\right)\left\{H_{n}(x)-H_{n-m}(x)\right\} \quad(m \leq n) .
$$

The binomial coefficients can be expressed as gamma function

$$
\left(\begin{array}{l}
s \\
t
\end{array}\right)=\frac{\Gamma(s+1)}{\Gamma(t+1) \Gamma(s-t+1)} \quad \text { for } \quad s, t \in \mathbb{C} \backslash \mathbb{Z}^{-} .
$$

The beta function $B(s, t)$, defined by

$$
B(s, t)=\int_{0}^{1} u^{s-1}(1-u)^{t-1} d u \quad(\mathfrak{R}(s)>0, \mathfrak{R}(t)>0),
$$

has a closed relationship with gamma function

$$
B(s, t)=\frac{\Gamma(s) \Gamma(t)}{\Gamma(s+t)} .
$$


There are many nice identities about harmonic numbers and binomial coefficients. For example, by making use of integral, it is not hard to derive (cf. [1, Lemma 1])

$$
\sum_{k=1}^{n} \frac{H_{k}}{k}=\frac{1}{2}\left[H_{n}^{2}+H_{n}^{\langle 2\rangle}\right]
$$

Recently, Batir [2] obtained a series of identities based on the binomial transformation formula

$$
\sum_{k=0}^{n}\left(\begin{array}{c}
s+n \\
k
\end{array}\right) x^{k}=(1+x)^{n}\left[1+s \sum_{k=0}^{n-1} \frac{\left(\begin{array}{c}
s+k \\
k
\end{array}\right)}{k+1}\left(\frac{x}{1+x}\right)^{k+1}\right] .
$$

Throughout the paper, we use the rising and falling factorials, defined by

$$
(x)_{0}=1 \quad \text { and } \quad(x)_{n}=x(x+1) \cdots(x+n-1) \quad \text { for } n \in \mathbb{N},
$$

and

$$
\langle x\rangle_{0}=1 \quad \text { and } \quad\langle x\rangle_{n}=x(x-1) \cdots(x-n+1) \quad \text { for } \quad n \in \mathbb{N} .
$$

The rest of the paper is organized as follows. In the next section, we firstly give a proof of Lemma 2.1, which is equivalent to Batir's binomial relation (5), and then derive some consequences by integration and derivation method. The paper ends with the third section, where several identities are established.

\section{Main results}

The identity in the following lemma is equivalent to Batir's identity (5). Here, we present an elementary proof, instead of induction.

Lemma 2.1. For $n \in \mathbb{N}$, the following relation holds

$$
\sum_{k=0}^{n}\left(\begin{array}{c}
x+k \\
k
\end{array}\right) z^{k}=\sum_{k=0}^{n}\left(\begin{array}{c}
x+n+1 \\
k
\end{array}\right) z^{k}(1-z)^{n-k}
$$

Proof. Applying the Vandermonde convolution

$$
\sum_{i=0}^{k}\left(\begin{array}{c}
x+i \\
i
\end{array}\right)\left(\begin{array}{c}
n-i \\
k-i
\end{array}\right)=\left(\begin{array}{c}
x+n+1 \\
k
\end{array}\right)
$$

to the right side of (6) gives

$$
\begin{aligned}
\sum_{k=0}^{n}\left(\begin{array}{c}
x+n+1 \\
k
\end{array}\right) z^{k}(1-z)^{n-k} & =\sum_{k=0}^{n} \sum_{i=0}^{k}\left(\begin{array}{c}
x+i \\
i
\end{array}\right)\left(\begin{array}{c}
n-i \\
k-i
\end{array}\right) z^{k}(1-z)^{n-k} \\
& =\sum_{i=0}^{n}\left(\begin{array}{c}
x+i \\
i
\end{array}\right) z^{i} \sum_{k=i}^{n}\left(\begin{array}{c}
n-i \\
k-i
\end{array}\right) z^{k-i}(1-z)^{n-k} \\
& =\sum_{i=0}^{n}\left(\begin{array}{c}
x+i \\
i
\end{array}\right) z^{i}
\end{aligned}
$$

which completes the proof.

Letting $z=1$ in (6), we have the following well-known formula [7, P.159]

$$
\sum_{k=0}^{n}\left(\begin{array}{c}
x+k \\
k
\end{array}\right)=\left(\begin{array}{c}
x+n+1 \\
n
\end{array}\right) \text {. }
$$

\section{Corollary 2.1.}

$$
\sum_{k=0}^{n}\left(\begin{array}{c}
x+k \\
k
\end{array}\right) H_{k}(x)=\left(\begin{array}{c}
x+n+1 \\
n
\end{array}\right)\left\{H_{n+1}(x)-\frac{1}{1+x}\right\} .
$$

Proof. The proof follows from differentiating with respect to $x$ both sides of (7). 


\section{Corollary 2.2.}

$$
\sum_{k=0}^{n-1} \frac{\left(\begin{array}{c}
x+n+1 \\
k+1
\end{array}\right)}{(n-k)\left(\begin{array}{c}
n \\
k
\end{array}\right)}=H_{n}+\sum_{k=1}^{n} \frac{\left(\begin{array}{c}
x+k \\
k
\end{array}\right)}{k}
$$

Proof. Separating the term of $k=0$ from the right-hand sum of (6), and then replacing $k$ by $k+1$, we obtain

$$
\sum_{k=0}^{n}\left(\begin{array}{c}
x+k \\
k
\end{array}\right) z^{k}=(1-z)^{n}+\sum_{k=0}^{n-1}\left(\begin{array}{c}
x+n+1 \\
k+1
\end{array}\right) z^{k+1}(1-z)^{n-k-1} .
$$

Putting the first term of the sum on the left side to the right, and then dividing the resulting equation by $z$, we have

$$
\sum_{k=1}^{n}\left(\begin{array}{c}
x+k \\
k
\end{array}\right) z^{k-1}=\frac{(1-z)^{n}-1}{z}+\sum_{k=0}^{n-1}\left(\begin{array}{c}
x+n+1 \\
k+1
\end{array}\right) z^{k}(1-z)^{n-k-1} .
$$

Integrating from $z=0$ to $z=1$, we get

$$
\sum_{k=1}^{n}\left(\begin{array}{c}
x+k \\
k
\end{array}\right) \frac{1}{k}=-\int_{0}^{1} \frac{(1-z)^{n}-1}{(1-z)-1} d z+\sum_{k=0}^{n-1}\left(\begin{array}{c}
x+n+1 \\
k+1
\end{array}\right) \int_{0}^{1} z^{k}(1-z)^{n-k-1} d z .
$$

According to

$$
\int_{0}^{1} \frac{(1-z)^{n}-1}{(1-z)-1} d z=H_{n} \quad \text { and } \quad \int_{0}^{1} z^{k}(1-z)^{n-k-1} d z=B(k+1, n-k)=\frac{1}{(n-k)\left(\begin{array}{l}
n \\
k
\end{array}\right)}
$$

we complete the proof.

\section{Corollary 2.3.}

$$
\sum_{k=0}^{n-1} \frac{\left(\begin{array}{c}
x+n+1 \\
k+1
\end{array}\right)}{(n-k)\left(\begin{array}{l}
n \\
k
\end{array}\right)} H_{k}=\frac{H_{n}^{2}-H_{n}^{\langle 2\rangle}}{2}+\sum_{k=1}^{n} \frac{\left(\begin{array}{c}
x+k \\
k
\end{array}\right)}{k^{2}}\left(k H_{n}-1\right) .
$$

Proof. Integrating both sides of (10) from $z=0$ to $z=u$, we get

$$
\sum_{k=1}^{n}\left(\begin{array}{c}
x+k \\
k
\end{array}\right) \frac{u^{k}}{k}=\int_{0}^{u} \frac{(1-z)^{n}-1}{z} d z+\sum_{k=0}^{n-1}\left(\begin{array}{c}
x+n+1 \\
k+1
\end{array}\right) \int_{0}^{u} z^{k}(1-z)^{n-k-1} d z .
$$

Integrating again the above equation from $u=0$ to $u=1$, after dividing by $u$, we find that

$$
\sum_{k=1}^{n}\left(\begin{array}{c}
x+k \\
k
\end{array}\right) \frac{1}{k^{2}}=\int_{0}^{1} \frac{1}{u} \int_{0}^{u} \frac{(1-z)^{n}-1}{z} d z d u+\sum_{k=0}^{n-1}\left(\begin{array}{c}
x+n+1 \\
k+1
\end{array}\right) \int_{0}^{1} \frac{1}{u} \int_{0}^{u} z^{k}(1-z)^{n-k-1} d z d u .
$$

By means of integration by parts, we obtain

$$
\begin{aligned}
\int_{0}^{1} \frac{1}{u} \int_{0}^{u} \frac{(1-z)^{n}-1}{z} d z d u & =-\left.\frac{n}{2} \frac{d^{2}}{d t^{2}} \int_{0}^{1} u^{t}(1-u)^{n-1} d u\right|_{t=0}=-\left.\frac{n}{2} \frac{d^{2}}{d t^{2}} \frac{\Gamma(t+1) \Gamma(n)}{\Gamma(t+n+1)}\right|_{t=0} \\
& =-\frac{H_{n}^{2}+H_{n}^{\langle 2\rangle}}{2}
\end{aligned}
$$

and

$$
\begin{aligned}
\int_{0}^{1} \frac{1}{u} \int_{0}^{u} z^{k}(1-z)^{n-k-1} d z d u & =-\left.\frac{d}{d t} \int_{0}^{1} u^{t}(1-u)^{n-k-1} d u\right|_{t=k}=-\left.\frac{d}{d t} \frac{\Gamma(t+1) \Gamma(n-k)}{\Gamma(n+t-k+1)}\right|_{t=k} \\
& =\frac{H_{n}-H_{k}}{(n-k)\left(\begin{array}{c}
n \\
k
\end{array}\right)} .
\end{aligned}
$$

Applying (9), we complete the proof.

\section{Corollary 2.4 .}

$$
\sum_{k=0}^{n-1} \frac{\left(\begin{array}{c}
x+n+1 \\
k+1
\end{array}\right)}{(n-k)\left(\begin{array}{c}
n \\
k
\end{array}\right)}\left[H_{n+1}(x)-H_{n-k}(x)\right]=\sum_{k=1}^{n} \frac{\left(\begin{array}{c}
x+k \\
k
\end{array}\right)}{k} H_{k}(x) .
$$

Proof. The proof follows from differentiating with respect to $x$ both sides of (9). 


\section{Corollary 2.5.}

$$
\sum_{k=0}^{n-1} \frac{\left(\begin{array}{c}
x+n+1 \\
k+1
\end{array}\right)}{(n-k)\left(\begin{array}{c}
n \\
k
\end{array}\right)}\left[H_{n+1}(x)-H_{n-k}(x)\right] H_{k}=\sum_{k=1}^{n} \frac{\left(\begin{array}{c}
x+k \\
k
\end{array}\right)}{k^{2}}\left(k H_{n}-1\right) H_{k}(x) .
$$

Proof. The proof follows from differentiating with respect to $x$ both sides of (11).

\section{Corollary 2.6.}

$$
\sum_{k=0}^{n} k\left(\begin{array}{c}
x+k \\
k
\end{array}\right)=(x+1)\left(\begin{array}{c}
x+n+1 \\
n-1
\end{array}\right)
$$

Proof. The proof follows by differentiating with respect to $z$ both sides of (6), and then letting $z=1$.

\section{Corollary 2.7.}

$$
\sum_{k=0}^{n} k\left(\begin{array}{c}
x+k \\
k
\end{array}\right) H_{k}(x)=(x+1)\left(\begin{array}{c}
x+n+1 \\
n-1
\end{array}\right)\left\{H_{n+1}(x)-\frac{1}{x+2}\right\}
$$

Proof. The result can be obtained by deriving $x$ for the identity in Corollary 2.6.

\section{Identities}

Based on the results obtained in Section 2, we can establish several interesting identities by taking special values of the parameter $x$, including certain well-known identities.

For example, letting $x=-\frac{1}{2}$ in (7) and $x=0$ in (8), we recover the two well-known identities

$$
\sum_{k=0}^{n} \frac{\left(\begin{array}{c}
2 k \\
k
\end{array}\right)}{4^{k}}=\frac{2 n+1}{4^{n}}\left(\begin{array}{c}
2 n \\
n
\end{array}\right) \text { and } \sum_{k=0}^{n} H_{k}=(n+1)\left(H_{n+1}-1\right) .
$$

Further examples can analogously be derived as follows.

Identity 3.1. For $n \in \mathbb{N}_{0}, m \in \mathbb{N}_{0} \cup\{-1\}$, it holds that

$$
\sum_{k=0}^{n} \frac{\left(\begin{array}{c}
2 m+2 k+2 \\
m+k+1
\end{array}\right)\left(\begin{array}{c}
m+k+1 \\
k
\end{array}\right)}{4^{k}}=\frac{\left(\begin{array}{c}
2 m+2 n+4 \\
m+n+2
\end{array}\right)\left(\begin{array}{c}
m+n+2 \\
m+2
\end{array}\right)\left(\begin{array}{c}
2 m+2 \\
m+1
\end{array}\right)}{4^{n}\left(\begin{array}{c}
2 m+4 \\
m+2
\end{array}\right)} .
$$

Proof. Letting $x=\frac{1}{2}+m\left(m \in \mathbb{N}_{0} \cup\{-1\}\right)$ in (7), we have

$$
\sum_{k=0}^{n}\left(\begin{array}{c}
m+k+\frac{1}{2} \\
k
\end{array}\right)=\left(\begin{array}{c}
m+n+\frac{3}{2} \\
n
\end{array}\right) .
$$

Then the identity follows by

$$
\left(\begin{array}{c}
m+k+\frac{1}{2} \\
k
\end{array}\right)=\frac{\left\langle m+k+\frac{1}{2}\right\rangle_{k}}{k !}=\frac{\left(\begin{array}{c}
2(m+k+1) \\
m+k+1
\end{array}\right)\left(\begin{array}{c}
m+k+1 \\
k
\end{array}\right)}{2^{2 k}\left(\begin{array}{c}
2 m+2 \\
m+1
\end{array}\right)},
$$

and

$$
\left(\begin{array}{c}
m+n+\frac{3}{2} \\
n
\end{array}\right)=\frac{\left\langle m+n+\frac{3}{2}\right\rangle_{n}}{n !}=\frac{\left(\begin{array}{c}
(m+n+2) \\
m+n+2
\end{array}\right)\left(\begin{array}{c}
m+n+2 \\
m+2
\end{array}\right)}{2^{2 n}\left(\begin{array}{c}
2 m+4 \\
m+2
\end{array}\right)} .
$$

Identity 3.2. For $m, n \in \mathbb{N}_{0}$, it holds that

$$
\sum_{k=0}^{n} \frac{(-1)^{k}\left(\begin{array}{c}
m+n+1 \\
k
\end{array}\right)}{4^{k}\left(\begin{array}{c}
2 m+2 n-2 k+2 \\
m+n-k+1
\end{array}\right)}=(-1)^{n} \frac{\left(\begin{array}{c}
2 m+2 n \\
m+n
\end{array}\right)\left(\begin{array}{c}
m+n \\
n
\end{array}\right)}{4^{n}\left(\begin{array}{c}
2 m+2 n+2 \\
m+n+1
\end{array}\right)} .
$$

Proof. Replacing $x$ by $x-n-1$ in (7), we have

$$
\sum_{k=0}^{n}\left(\begin{array}{c}
x+k-n-1 \\
k
\end{array}\right)=\left(\begin{array}{l}
x \\
n
\end{array}\right) .
$$


Taking $x=-\frac{1}{2}-m\left(m \in \mathbb{N}_{0}\right)$ in (18), we get

$$
\sum_{k=0}^{n}\left(\begin{array}{c}
-m-n+k-\frac{3}{2} \\
k
\end{array}\right)=\left(\begin{array}{c}
-m-\frac{1}{2} \\
n
\end{array}\right) .
$$

Noticing that

$$
\left(\begin{array}{c}
-m-n+k-\frac{3}{2} \\
k
\end{array}\right)=(-1)^{k}\left(\begin{array}{c}
m+n+\frac{1}{2} \\
k
\end{array}\right)=(-1)^{k} \frac{\left(\begin{array}{c}
2 m+2 n+2 \\
m+n+1
\end{array}\right)\left(\begin{array}{c}
m+n+1 \\
k
\end{array}\right)}{4^{k}\left(\begin{array}{c}
2 m+2 n-2 k+2 \\
m+n-k+1
\end{array}\right)},
$$

and

we get the desired identity.

$$
\left(\begin{array}{c}
-m-\frac{1}{2} \\
n
\end{array}\right)=(-1)^{n}\left(\begin{array}{c}
m+n-\frac{1}{2} \\
n
\end{array}\right)=(-1)^{n} \frac{\left(\begin{array}{c}
2 m+2 n \\
m+n
\end{array}\right)\left(\begin{array}{c}
m+n \\
n
\end{array}\right)}{4^{n}},
$$

For $m=0$ in (17), we have the following identity

$$
\sum_{k=0}^{n} \frac{(-1)^{k}\left(\begin{array}{c}
n+1 \\
k
\end{array}\right)}{4^{k}\left(\begin{array}{c}
2 n-2 k+2 \\
n-k+1
\end{array}\right)}=(-1)^{n} \frac{n+1}{2^{2 n+1}(2 n+1)} .
$$

Identity 3.3. For $n \in \mathbb{N}_{0}, m \in \mathbb{N}_{0} \cup\{-1\}$, it holds that

$$
\begin{aligned}
\sum_{k=0}^{n} & \frac{\left(\begin{array}{c}
2 m+2 k+2 \\
m+k+1
\end{array}\right)\left(\begin{array}{c}
m+k+1 \\
k
\end{array}\right)}{4^{k}}\left\{2 H_{2 m+2 k+1}-H_{m+k}\right\} \\
\quad= & \frac{\left(\begin{array}{c}
2 m+2 n+4 \\
m+n+2
\end{array}\right)\left(\begin{array}{c}
m+n+2 \\
m+2
\end{array}\right)\left(\begin{array}{c}
2 m+2 \\
m+1
\end{array}\right)}{4^{n}\left(\begin{array}{c}
2 m+4 \\
m+2
\end{array}\right)}\left\{2 H_{2 m+2 n+3}-H_{m+n+1}-\frac{2}{3+2 m}\right\} .
\end{aligned}
$$

Proof. Setting $x=\frac{1}{2}+m\left(m \in \mathbb{N}_{0} \cup\{-1\}\right)$ in (8), we get (20) with the help of (16).

If setting $m=-1,0$ in (20), we get, respectively, the following two identities

$$
\begin{aligned}
& \sum_{k=0}^{n} \frac{\left(\begin{array}{c}
2 k \\
k
\end{array}\right)}{4^{k}}\left\{2 H_{2 k-1}-H_{k-1}\right\}=\frac{2 n+1}{4^{n}}\left(\begin{array}{c}
2 n \\
n
\end{array}\right)\left\{2 H_{2 n+1}-H_{n}-2\right\}, \\
& \sum_{k=0}^{n} \frac{(k+1)\left(\begin{array}{c}
2 k+2 \\
k+1
\end{array}\right)}{4^{k}}\left\{2 H_{2 k+1}-H_{k}\right\}=\frac{(n+1)(n+2)}{6} \frac{\left(\begin{array}{c}
2 n+4 \\
n+2
\end{array}\right)}{4^{n}}\left\{2 H_{2 n+3}-H_{n+1}-\frac{2}{3}\right\} .
\end{aligned}
$$

Identity 3.4. For a nonnegative integer $m$, we have

$$
\sum_{k=0}^{n-1} \frac{\left(\begin{array}{c}
n+m+1 \\
k+1
\end{array}\right)}{4^{k+1}(n-k)\left(\begin{array}{c}
n \\
k
\end{array}\right)\left(\begin{array}{c}
2 n+2 m-2 k \\
n+m-k
\end{array}\right)}=\frac{1}{\left(\begin{array}{c}
2 n+2 m+2 \\
n+m+1
\end{array}\right)}\left\{H_{n}+\frac{1}{\left(\begin{array}{c}
2 m \\
m
\end{array}\right)} \sum_{k=1}^{n} \frac{\left(\begin{array}{c}
k+m \\
k
\end{array}\right)\left(\begin{array}{c}
2 k+2 m \\
k+m
\end{array}\right)}{4^{k} k}\right\} .
$$

Proof. Taking $x=m-\frac{1}{2}$ in (9), we obtain

$$
\sum_{k=0}^{n-1} \frac{\left(\begin{array}{c}
n+m+\frac{1}{2} \\
k+1
\end{array}\right)}{(n-k)\left(\begin{array}{l}
n \\
k
\end{array}\right)}=H_{n}+\sum_{k=1}^{n} \frac{\left(\begin{array}{c}
k+m-\frac{1}{2} \\
k
\end{array}\right)}{k}
$$

In view of

$$
\left(\begin{array}{c}
n+m+\frac{1}{2} \\
k+1
\end{array}\right)=\frac{\left\langle n+m+\frac{1}{2}\right\rangle_{k+1}}{(k+1) !}=\frac{\left(\begin{array}{c}
2 n+2 m+2 \\
n+m+1
\end{array}\right)\left(\begin{array}{c}
n+m+1 \\
k+1
\end{array}\right)}{4^{k+1}\left(\begin{array}{c}
2 n+2 m-2 k \\
n+m-k
\end{array}\right)},
$$

and

$$
\left(\begin{array}{c}
k+m-\frac{1}{2} \\
k
\end{array}\right)=\frac{\left\langle k+m-\frac{1}{2}\right\rangle_{k}}{k !}=\frac{\left(\begin{array}{c}
k+m \\
k
\end{array}\right)\left(\begin{array}{c}
2 k+2 m \\
k+m
\end{array}\right)}{4^{k}\left(\begin{array}{c}
2 m \\
m
\end{array}\right)},
$$

we complete the proof.

Two special cases corresponding to $m=1,2$ in (23) are recorded below

$$
\begin{aligned}
& \sum_{k=0}^{n-1} \frac{1}{4^{k+1}(k+1)(n-k)\left(\begin{array}{c}
2 n-2 k \\
n-k
\end{array}\right)}=\frac{1}{(n+1)\left(\begin{array}{c}
2 n+2 \\
n+1
\end{array}\right)}\left\{H_{n}+\sum_{k=1}^{n} \frac{\left(\begin{array}{c}
2 k \\
k
\end{array}\right)}{4^{k} k}\right\}, \\
& \sum_{k=0}^{n-1} \frac{1}{4^{k+1}(k+1)(n-k)_{2}\left(\begin{array}{c}
2 n+2-2 k \\
n+1-k
\end{array}\right)}=\frac{1}{(n+1)_{2}\left(\begin{array}{c}
2 n+4 \\
n+2
\end{array}\right)}\left\{H_{n}+\frac{1}{2} \sum_{k=1}^{n} \frac{(k+1)\left(\begin{array}{c}
2 k+2 \\
k+1
\end{array}\right)}{4^{k} k}\right\} .
\end{aligned}
$$


Identity 3.5. For $n \in \mathbb{N}$, it holds that

$$
\sum_{k=0}^{n-1} \frac{1}{4^{k+1}(k+1)\left(\begin{array}{c}
2 n-2 k-2 \\
n-k-1
\end{array}\right)}=\frac{1}{\left(\begin{array}{c}
2 n \\
n
\end{array}\right)}\left\{H_{n}-\sum_{k=1}^{n} \frac{\left(\begin{array}{c}
2 k \\
k
\end{array}\right)}{4^{k} k(2 k-1)}\right\} .
$$

Proof. Taking $x=-\frac{3}{2}$ in (9), we obtain

$$
\sum_{k=0}^{n-1} \frac{\left(\begin{array}{c}
n-\frac{1}{2} \\
k+1
\end{array}\right)}{(n-k)\left(\begin{array}{l}
n \\
k
\end{array}\right)}=H_{n}+\sum_{k=1}^{n} \frac{\left(\begin{array}{c}
k-\frac{3}{2} \\
k
\end{array}\right)}{k}
$$

Since

$$
\left(\begin{array}{c}
n-\frac{1}{2} \\
k+1
\end{array}\right)=\frac{\left(\begin{array}{c}
2 n \\
n
\end{array}\right)\left(\begin{array}{c}
n \\
k+1
\end{array}\right)}{4^{k+1}\left(\begin{array}{c}
2 n-2 k-2 \\
n-k-1
\end{array}\right)} \quad \text { and } \quad\left(\begin{array}{c}
k-\frac{3}{2} \\
k
\end{array}\right)=-\frac{\left(\begin{array}{c}
2 k \\
k
\end{array}\right)}{4^{k}(2 k-1)}
$$

this completes the proof.

Identity 3.6. For a positive integer $m$, we have

$$
\sum_{k=0}^{n-1} \frac{H_{k}}{(k+1)(n-k)_{m}}=\frac{1}{(n+1)_{m}}\left\{\frac{H_{n}^{2}-H_{n}^{\langle 2\rangle}}{2}+\sum_{k=1}^{n} \frac{(m)_{k}}{k^{2} k !}\left(k H_{n}-1\right)\right\}
$$

Proof. Let $x=m-1$ in (11), we get

$$
\sum_{k=0}^{n-1} \frac{\left(\begin{array}{c}
m+n \\
k+1
\end{array}\right)}{(n-k)\left(\begin{array}{l}
n \\
k
\end{array}\right)} H_{k}=\frac{H_{n}^{2}-H_{n}^{\langle 2\rangle}}{2}+\sum_{k=1}^{n}\left(\begin{array}{c}
m+k-1 \\
k
\end{array}\right)\left(\frac{H_{n}}{k}-\frac{1}{k^{2}}\right) .
$$

In view of

$$
\left(\begin{array}{c}
m+k-1 \\
k
\end{array}\right)=\frac{(m)_{k}}{k !} \text { and } \quad \frac{\left(\begin{array}{c}
m+n \\
k+1
\end{array}\right)}{(n-k)\left(\begin{array}{l}
n \\
k
\end{array}\right)}=\frac{(n+1)_{m}}{(k+1)(n-k)_{m}}
$$

this completes the proof.

Taking $m=1$ in (27), we have the following special case

$$
\sum_{k=0}^{n-1} \frac{H_{k}}{(k+1)(n-k)}=\frac{3}{2(n+1)}\left\{H_{n}^{2}-H_{n}^{\langle 2\rangle}\right\}
$$

Identity 3.7. For a nonnegative integer $m$, we have

$$
\sum_{k=0}^{n-1} \frac{\left(\begin{array}{c}
n+m+1 \\
k+1
\end{array}\right)}{4^{k+1}(n-k)\left(\begin{array}{c}
n \\
k
\end{array}\right)\left(\begin{array}{c}
2 n+2 m-2 k \\
n+m-k
\end{array}\right)} H_{k}=\frac{1}{\left(\begin{array}{c}
2 n+2 m+2 \\
n+m+1
\end{array}\right)}\left\{\frac{H_{n}^{2}-H_{n}^{\langle 2\rangle}}{2}+\frac{1}{\left(\begin{array}{c}
2 m \\
m
\end{array}\right)} \sum_{k=1}^{n} \frac{\left(\begin{array}{c}
k+m \\
k
\end{array}\right)\left(\begin{array}{c}
2 k+2 m \\
k+m
\end{array}\right)}{4^{k} k^{2}}\left(k H_{n}-1\right)\right\} .
$$

Proof. Letting $x=m-\frac{1}{2}$ in (11), we get

$$
\sum_{k=0}^{n-1} \frac{\left(\begin{array}{c}
n+m+\frac{1}{2} \\
k+1
\end{array}\right)}{(n-k)\left(\begin{array}{l}
n \\
k
\end{array}\right)} H_{k}=\frac{H_{n}^{2}-H_{n}^{\langle 2\rangle}}{2}+\sum_{k=1}^{n} \frac{\left(\begin{array}{c}
k+m-\frac{1}{2} \\
k
\end{array}\right)}{k^{2}}\left(k H_{n}-1\right) .
$$

Since

$$
\left(\begin{array}{c}
k+m-\frac{1}{2} \\
k
\end{array}\right)=\frac{\left(\begin{array}{c}
k+m \\
k
\end{array}\right)\left(\begin{array}{c}
2 k+2 m \\
k+m
\end{array}\right)}{4^{k}\left(\begin{array}{c}
2 m \\
m
\end{array}\right)} \quad \text { and } \quad\left(\begin{array}{c}
n+m+\frac{1}{2} \\
k+1
\end{array}\right)=\frac{\left(\begin{array}{c}
2 n+2 m+2 \\
n+m+1
\end{array}\right)\left(\begin{array}{c}
n+m+1 \\
k+1
\end{array}\right)}{2^{2 k+2}\left(\begin{array}{c}
2 n+2 m-2 k \\
n+m-k
\end{array}\right)}
$$

the proof can be completed.

When $m=0,(29)$ reduces to the following identity

$$
\sum_{k=0}^{n-1} \frac{H_{k}}{4^{k+1}(k+1)(n-k)\left(\begin{array}{c}
2 n-2 k \\
n-k
\end{array}\right)}=\frac{1}{(n+1)\left(\begin{array}{c}
2 n+2 \\
n+1
\end{array}\right)}\left\{\frac{H_{n}^{2}-H_{n}^{\langle 2\rangle}}{2}+\sum_{k=1}^{n} \frac{\left(\begin{array}{c}
2 k \\
k
\end{array}\right)}{4^{k} k^{2}}\left(k H_{n}-1\right)\right\} .
$$

Identity 3.8. For a nonnegative integer $m$, holds the identity

$$
\sum_{k=0}^{n-1} \frac{H_{m+n-k}}{(k+1)(n-k)_{m+1}}=\frac{1}{(n+1)_{m+1}}\left\{H_{n} H_{m+n+1}+\sum_{k=1}^{n} \frac{(m+1)_{k}}{k k !}\left(H_{m+n+1}+H_{m}-H_{k+m}\right)\right\} .
$$


Proof. Letting $x=m\left(m \in \mathbb{N}_{0}\right)$ in (12), we get

$$
\sum_{k=0}^{n-1} \frac{\left(\begin{array}{c}
m+n+1 \\
k+1
\end{array}\right)}{(n-k)\left(\begin{array}{c}
n \\
k
\end{array}\right)}\left\{H_{n+1}(x)-H_{n-k}(m)\right\}=\sum_{k=1}^{n} \frac{\left(\begin{array}{c}
m+k \\
k
\end{array}\right)}{k} H_{k}(m) .
$$

According to the formula

$$
H_{n}(m)=H_{m+n}-H_{m}
$$

and (9), we complete the proof.

For $m=0,1,(31)$ yields the following two special cases

$$
\begin{aligned}
& \sum_{k=0}^{n-1} \frac{H_{n-k}}{(k+1)(n-k)}=\frac{1}{2(n+1)}\left\{4 H_{n} H_{n+1}-H_{n}^{2}-H_{n}^{\langle 2\rangle}\right\}, \\
& \sum_{k=0}^{n-1} \frac{H_{n-k+1}}{(k+1)(n-k)_{2}}=\frac{1}{2(n+1)_{2}}\left\{4 H_{n} H_{n+2}+2 n\left(H_{n+2}+2\right)-2(n+1) H_{n}-H_{n}^{2}-H_{n}^{\langle 2\rangle}\right\} .
\end{aligned}
$$

Identity 3.9. For a nonnegative integer $m$, there holds the identity

$$
\begin{gathered}
\sum_{k=0}^{n-1} \frac{2 H_{2 m+2 n-2 k-1}-H_{m+n-k-1}}{4^{k+1}(k+1)(n-k)_{m+1}\left(\begin{array}{c}
2 m+2 n-2 k \\
m+n-k
\end{array}\right)}=\frac{\left(2 H_{2 m+2 n+1}-H_{m+n}\right) H_{n}}{(n+1)_{m+1}\left(\begin{array}{c}
2 m+2 n+2 \\
m+n+1
\end{array}\right)}+\frac{1}{(n+1)_{m+1}\left(\begin{array}{c}
2 m+2 n+2 \\
m+n+1
\end{array}\right)\left(\begin{array}{c}
2 m \\
m
\end{array}\right)} \times \\
\left\{\sum_{k=1}^{n} \frac{(m+1)_{k}\left(\begin{array}{c}
2 m+2 k \\
m+k
\end{array}\right)}{4^{k} k k !}\left(2 H_{2 m+2 n+1}+2 H_{2 m}-H_{m+n}-H_{m}+H_{m+k-1}-2 H_{2 m+2 k-1}\right)\right\} .
\end{gathered}
$$

Proof. Taking $x=-\frac{1}{2}+m\left(m \in \mathbb{N}_{0}\right)$ in (12), we have

$$
\sum_{k=0}^{n-1} \frac{\left(\begin{array}{c}
m+n+\frac{1}{2} \\
k+1
\end{array}\right)}{(n-k)\left(\begin{array}{l}
n \\
k
\end{array}\right)}\left\{H_{n+1}\left(m-\frac{1}{2}\right)-H_{n-k}\left(m-\frac{1}{2}\right)\right\}=\sum_{k=1}^{n} \frac{\left(\begin{array}{c}
m+k-\frac{1}{2} \\
k
\end{array}\right)}{k} H_{k}(m) .
$$

By combining the method of proving (23) and the formula

$$
H_{n}\left(m-\frac{1}{2}\right)=2 H_{2 m+2 n-1}-H_{m+n-1}+H_{m}-2 H_{2 m},
$$

we can confirm the identity (34).

A special case of (34) with $m=0$ reads as

$$
\sum_{k=0}^{n-1} \frac{2 H_{2 n-2 k-1}-H_{n-k-1}}{4^{k+1}(k+1)(n-k)\left(\begin{array}{c}
2 n-2 k \\
n-k
\end{array}\right)}=\frac{H_{n}\left(2 H_{2 n+1}-H_{n}\right)}{(n+1)\left(\begin{array}{c}
2 n+2 \\
n+1
\end{array}\right)}+\frac{1}{(n+1)\left(\begin{array}{c}
2 n+2 \\
n+1
\end{array}\right)} \sum_{k=1}^{n} \frac{\left(\begin{array}{c}
2 k \\
k
\end{array}\right)}{4^{k} k}\left\{2 H_{2 n+1}-H_{n}-2 H_{2 k-1}+H_{k-1}\right\} .
$$

Identity 3.10. For a nonnegative integer $m$, we have

$$
\sum_{k=0}^{n-1} \frac{H_{k} H_{m+n-k}}{(k+1)(n-k)_{m+1}}=\frac{H_{n+m+1}\left[H_{n}^{2}-H_{n}^{\langle 2\rangle}\right]}{2(n+1)_{m+1}}+\frac{1}{(n+1)_{m+1}} \sum_{k=1}^{n} \frac{(m+1)_{k}}{k^{2} k !}\left(k H_{n}-1\right)\left\{H_{m+n+1}+H_{m}-H_{m+k}\right\} .
$$

Proof. Letting $x=m$ in (13), we can derive (36) with an aid of (11).

In particular, when $m=0$, we get from (36)

$$
\sum_{k=0}^{n-1} \frac{H_{k} H_{n-k}}{(k+1)(n-k)}=\frac{3 H_{n+1}\left[H_{n}^{2}-H_{n}^{\langle 2\rangle}\right]-H_{n}\left[H_{n}^{2}+H_{n}^{\langle 2\rangle}\right]}{2(n+1)}+\frac{1}{n+1} \sum_{k=1}^{n} \frac{H_{k}}{k^{2}} .
$$

Identity 3.11. For a nonnegative integer $m$, there holds the identity

$$
\begin{gathered}
\sum_{k=0}^{n-1} \frac{\left(2 H_{2 m+2 n-2 k-1}-H_{m+n-k-1}\right) H_{k}}{4^{k+1}(k+1)(n-k)_{m+1}\left(\begin{array}{c}
2 m+2 n-2 k \\
m+n-k
\end{array}\right)}=\frac{\left(2 H_{2 m+2 n+1}-H_{m+n}\right)\left[H_{n}^{2}-H_{n}^{\langle 2\rangle}\right]}{2(n+1)_{m+1}\left(\begin{array}{c}
2 m+2 n+2 \\
m+n+1
\end{array}\right)}+ \\
\frac{1}{(n+1)_{m+1}\left(\begin{array}{c}
2 m \\
m
\end{array}\right)\left(\begin{array}{c}
2 m+2 n+2 \\
m+n+1
\end{array}\right)} \sum_{k=1}^{n} \frac{(m+1)_{k}\left(\begin{array}{c}
2 m+2 k \\
m+k
\end{array}\right)}{4^{k} k^{2} k !}\left(k H_{n}-1\right)\left\{2 H_{2 m+2 n+1}+\right. \\
\left.2 H_{2 m}-H_{m+n}-H_{m}+H_{m+k-1}-2 H_{2 m+2 k-1}\right\} .
\end{gathered}
$$


Proof. The identity follows by taking $x=-\frac{1}{2}+m$ in (13) and using (29).

A special case of (38) corresponding to $m=0$ is recorded below

$$
\begin{aligned}
& \sum_{k=0}^{n-1} \frac{\left(2 H_{2 n-2 k-1}-H_{n-k-1}\right) H_{k}}{4^{k+1}(k+1)(n-k)\left(\begin{array}{c}
2 n-2 k \\
n-k
\end{array}\right)}=\frac{\left(2 H_{2 n+1}-H_{n}\right)\left[H_{n}^{2}-H_{n}^{\langle 2\rangle}\right]}{2(n+1)\left(\begin{array}{c}
2 n+2 \\
n+1
\end{array}\right)}+ \\
& \frac{1}{(n+1)\left(\begin{array}{c}
2 n+2 \\
n+1
\end{array}\right)} \sum_{k=1}^{n} \frac{\left(\begin{array}{c}
2 k \\
k
\end{array}\right)}{4^{k} k^{2}}\left(k H_{n}-1\right)\left\{2 H_{2 n+1}-H_{n}-2 H_{2 k-1}+H_{k-1}\right\} .
\end{aligned}
$$

Besides the identities derived above, by choosing other different special values of parameter $x$, it is possible to derive more finite series identities on harmonic numbers and binomial coefficients. The interested reader is encouraged to make further attempts.

\section{Acknowledgment}

The author is sincerely grateful to the anonymous referees for the helpful comments and valuable suggestions that contribute significantly to improving the manuscript.

\section{References}

[1] V. Adamchik, On Stirling numbers and Euler sums, J. Comput. Appl. Math. 79 (1997) 119-130.

[2] N. Batir, Combinatorial identities involving harmonic numbers, Integers 20 (2020) \#A25.

[3] J. Choi, Certain summation formulas involving harmonic numbers and generalized harmonic numbers, Appl. Math. Comput. 218 (2011) $734-740$.

[4] W. Chu, Partial-fraction decompositions and harmonic number identities, J. Combin. Math. Combin. Comput. 60 (2007) $139-153$.

[5] W. Chu, L. De Donno, Hypergeometric series and harmonic number identities, Adv. Appl. Math. 34 (2005) $123-137$.

[6] W. Chu, A. M. Fu, Dougall-Dixon formula and harmonic number identities, Ramanujan J. 18 (2009) 11-31.

[7] R. L. Graham, D. E. Knuth, O. Patashnik, Concrete Mathematics: A Foundation for Computer Science, China Machine Press, Beijing, 2002.

[8] A. Sofo, H. M. Srivastava, Identities for the harmonic numbers and binomial coefficients, Ramanujan J. 25 (2011) $93-113$. 